\title{
Improving Transmission Efficiency with Magnetic Coating Technology for Lightweight Wireless Power Transfer Coil Using Aluminum Plate
}

\author{
S. Endo, M. Sato, Y. Bu, and T. Mizuno \\ Department of Engineering, Shinshu University, 4-17-1 Wakasato, Nagano, Nagano, Japan
}

To improve the fuel efficiency of electric vehicles, it is necessary to reduce the weight of the wireless power transfer coil in the vehicles. Resistance due to the skin effect or the proximity effect increases during wireless power transfer, decreasing the transmission efficiency. This study aims to reduce the weight of the coil by replacing it with an aluminum plate coil, which is easy to manufacture and inexpensive. The weight of the coil was reduced by $3 / 4$ (from 1.9 to $0.44 \mathrm{~kg}$ ) when compared with copper Litz wire. Furthermore, the AC resistance was reduced by applying magnetic coating to the same coil. Consequently, the transmission efficiency increased from $88.2 \%$ to $89.3 \%$, an improvement of $1.1 \%$. The optimal material for magnetic coating was revealed in an analysis.

Keywords: wireless power transmission, aluminum plate, lightweight, low cost, high efficiency, copper loss reduction, magnetic composite material, magnetic coating technology

\section{Introduction}

As a power supply method for electric vehicles $(\mathrm{EVs})$, wireless power transmission is attracting attention because it allows to charge stationary EVs seamlessly and is not associated with cable-related problems such as forgetting to charge or leakage ${ }^{1-3}$. Wireless power transmission for EVs operates at a high frequency of $85 \mathrm{kHz}$. Since a high-frequency loss typically occurs in wireless power transfer coils ${ }^{4}$, the litz copper wire (LCW) with good high-frequency characteristics is generally used in wireless power transfer coils for $\mathrm{EVS}^{5-11)}$.

To improve the fuel efficiency of EVs, a weight reduction of the embedded coil is highly desirable. Furthermore, a coil that is inexpensive and easy to manufacture is required.

To meet these requirements, we examined a relatively facile fabrication of a coil using an aluminum plate. However, the aluminum plate (AP) coil has a large $\mathrm{AC}$ resistance due to the proximity effect ${ }^{12}$. At the same time, this resistance can be reduced by coating the coil with a magnetic layer ${ }^{13-15)}$. Therefore, we coated the AP coil with a magnetic composite material using a low-loss amorphous alloy powder achieving a magnetically coated plate (MCP) coil.

In this study, the optimal magnetic composite material was analyzed using the finite element method (FEM). Furthermore, the impedance characteristics of the coil were evaluated, and the transmission efficiencies of the AP and MCP coils were measured using the double-LCC resonant circuit. (a)

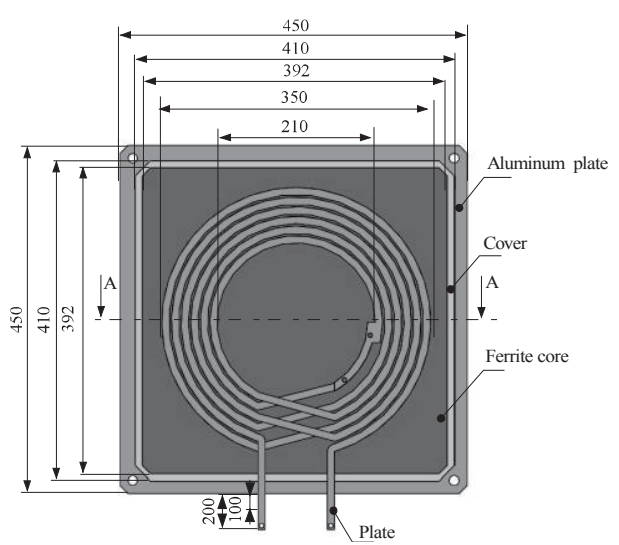

(b)

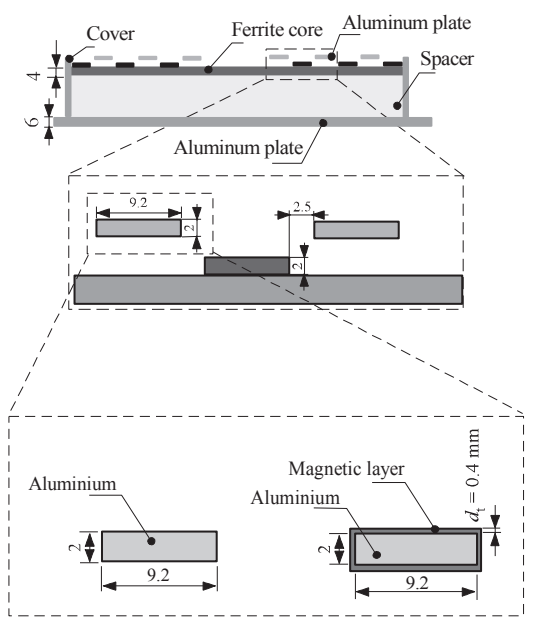

Fig. 1 Structure of the aluminum plate (AP) and magnetically coated plate (MCP) coils (unit: $\mathrm{mm}$ ): (a) plane view and (b) sectional view. (c) Structure of the AP and MCP. 


\section{Structure of the Wireless Power Transfer Coil}

\subsection{Structure of the aluminum plate coil}

Figure 1 illustrates the structure of the AP and MCP receiving-side coils in planar (Figure 1(a)) and sectional (Figure 1(b)) views. A ferrite core in the back yoke improves the inductance, while an AP on the back surface of the coil provides magnetic shielding ${ }^{16}$. Figure 1(c) depicts the structures of the AP and MCP used for the coil. The AP has a width of $9.2 \mathrm{~mm}$, a thickness of 2 $\mathrm{mm}$, and a conductor cross section of $A=18.4 \mathrm{~mm}^{2}$. A brush was used to apply the magnetic composite material to the AP, and the thickness of the magnetic layer of the resulting MCP was $0.4 \mathrm{~mm}$.

\subsection{Structure of the receiving-side litz copper wire coil}

Figures 2(a) and 2(b) illustrate the planar and sectional views of the LCW coil structure, respectively. The coil has a short spiral coil structure with an outer diameter of $350 \mathrm{~mm}$ and the number of turns $N=6$ wound in parallel.

(a)

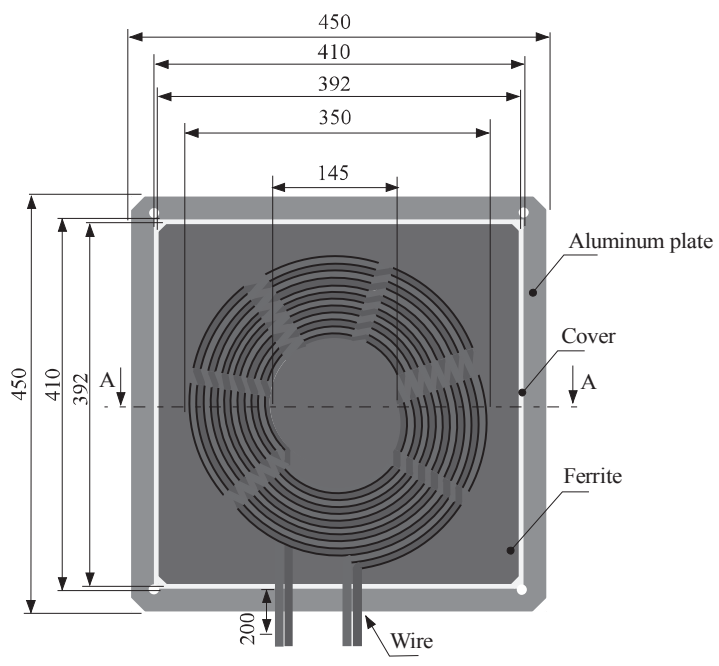

(b)

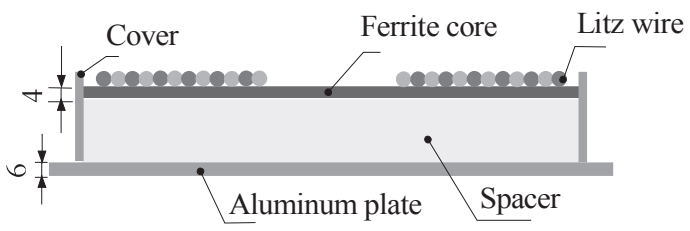

(c)

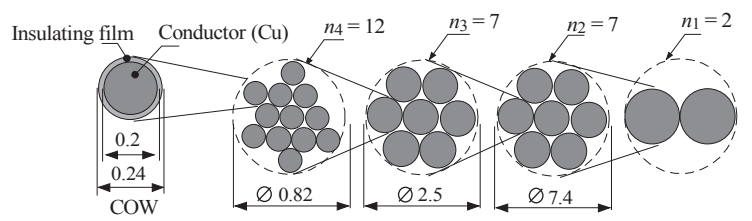

Fig. 2 Structure of the litz copper wire (LCW) coil (unit: $\mathrm{mm}$ ): (a) plane view and (b) sectional view. (c) Structure of the LCW.
Figure 2(c) illustrates the cross-sectional structure of the litz wire used in the coil. Generally, the LCW has a conductor diameter of $0.2 \mathrm{~mm}$ with a $0.02-\mathrm{mm}$ thick insulating film and a final diameter of $7.4 \mathrm{~mm}$. The number of strands $n$ was 1176 , while the conductor cross section was $A=36.8 \mathrm{~mm}^{2}$.

\subsection{Structure of the power transmission coil}

Figures 3(a) and 3(b) illustrate the planar and sectional views of the power transfer coil structure, respectively. The transfer coil used in this study, standardized by SAE, is a rectangular spiral coil containing the same litz wire as the LCW coil ${ }^{4}$.

Figure 3(c) illustrates the cross-sectional structure of the litz wire used in the coil. Generally, the LCW has a conductor diameter of $0.049 \mathrm{~mm}$ with a $5.5-\mu \mathrm{m}$ thick insulating film and a final diameter of $5.0 \mathrm{~mm}$. The number of strands $n$ was 4200 , and the conductor cross section was $A=7.92 \mathrm{~mm}^{2}$.

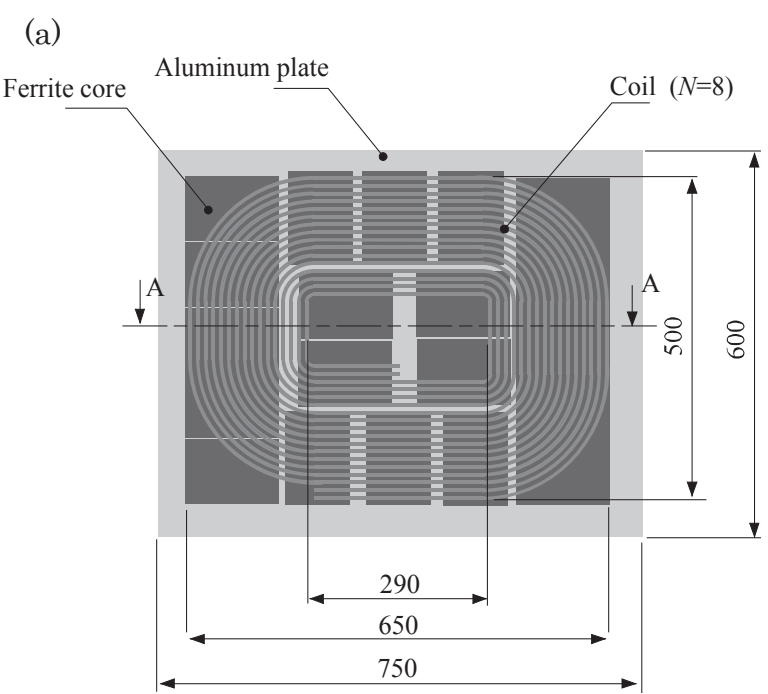

(b)
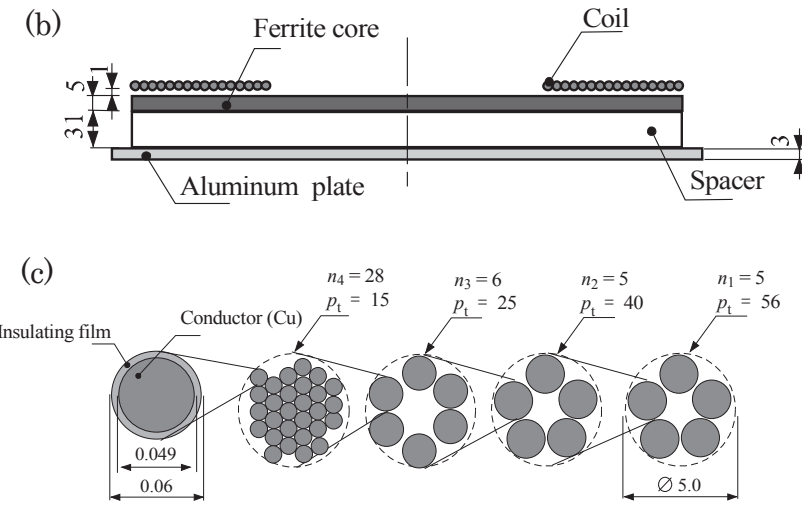

Fig. 3 Structure of power transmission coil (unit: $\mathrm{mm}$ ). (a) Plane view. (b) Section A-A.

(c) Wire structure of power transmission coil $\left(n=4200, A=7.9 \mathrm{~mm}^{2}\right)$. 


\section{Analysis of the Wireless Power Transfer Coil}

Figure 4 shows the results of the FEM-based analysis exhibiting the MCP coil characteristics with respect to permeability $(\mu)$. AC magnetic field analysis (Ansys Maxwell 2D) was performed, and the coil resistance $R$ and inductance $L$ were calculated. The quality factor $Q$ of the MCP coil was calculated according to Eq. (1). The transmission efficiency of the MCP coil $\eta_{\mathrm{C}}$ was calculated from the $k Q$ product using Eq. (2) ${ }^{17}$.

$$
\begin{aligned}
& Q=\frac{\omega L}{R} \\
& \eta_{\mathrm{c}}=\frac{(k Q)^{2}}{\left(1+\sqrt{1+(k Q)^{2}}\right)^{2}} \times 100 \quad(\%)
\end{aligned}
$$

Figure 4(a) depicts the trend associated with the resistance of the coil. As $\mu^{\prime}$ improves, the resistance decreases. This is because the induced magnetic flux of the magnetic layer of the coil is increased, while the proximity effect is reduced. Moreover, the resistance decreases due to the decrease in $\mu^{\prime \prime}$. This is because the iron loss decreased.

Figure 4(b) shows the inductance of the coil. An increase in $\mu^{\prime}$ improves the inductance, whereas no effect is observed due the variations in $\mu^{\prime \prime}$. As $\mu^{\prime}$ increases, the flux generated by the coil is confined inside the coil; hence, the flux linkage increases and the inductance improves. $\mu^{\prime \prime}$ does not affect the distribution of the magnetic flux or the inductance.

(a)

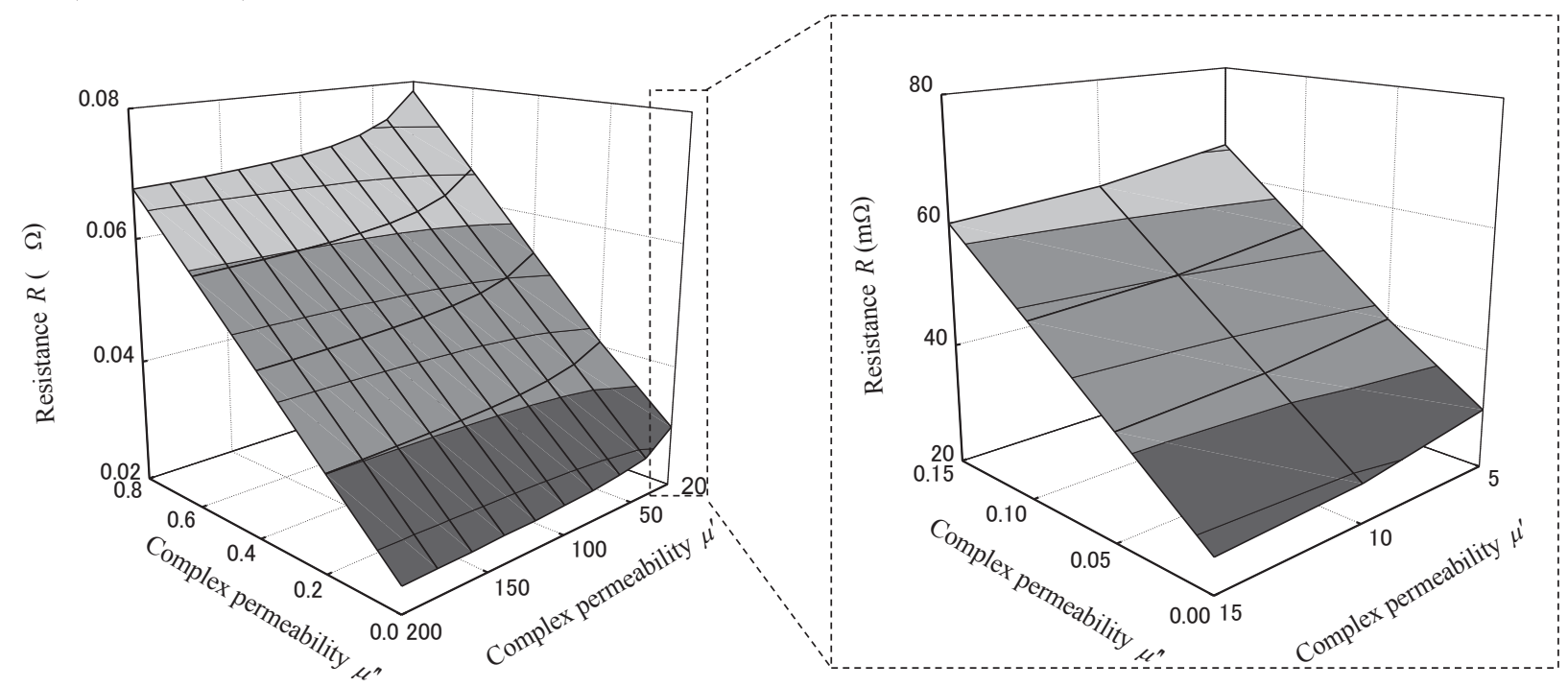

(b)

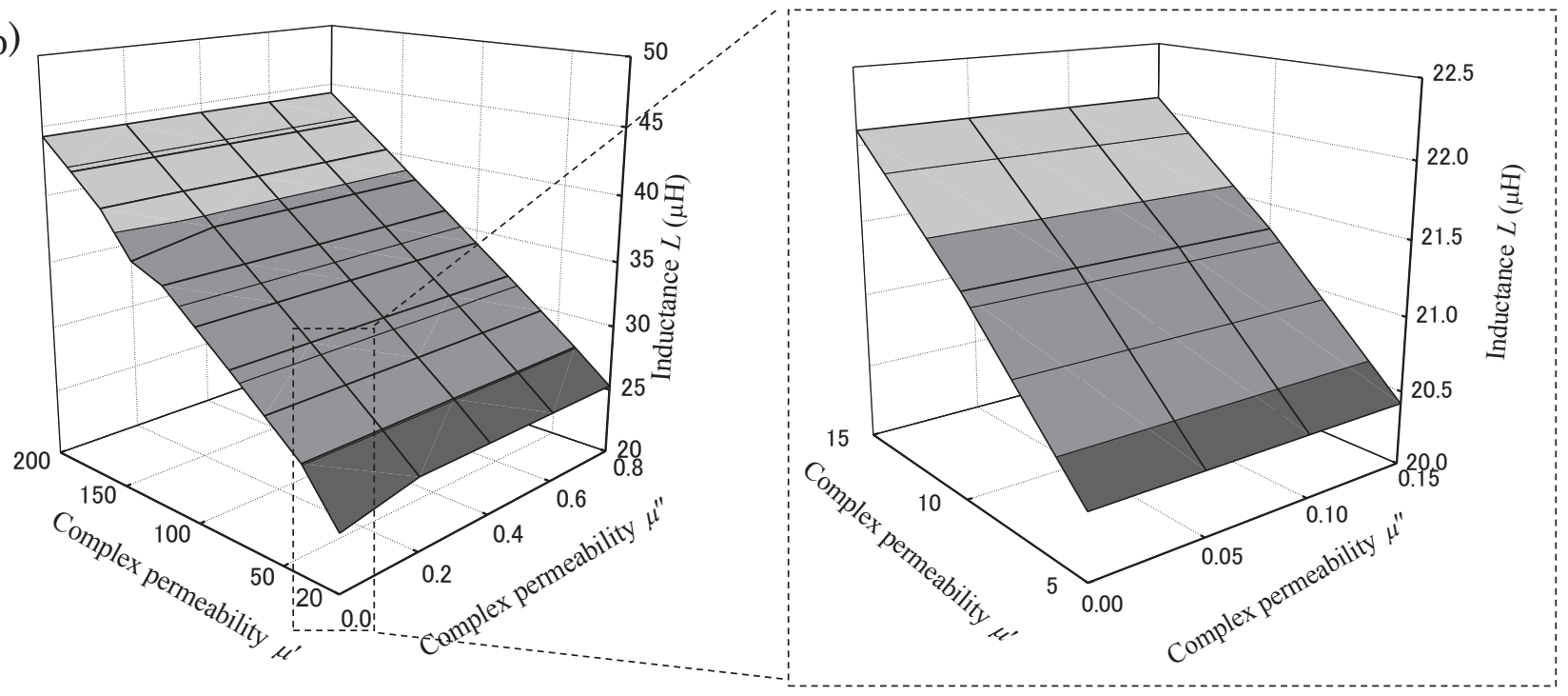

Fig. 4 Characteristics of the MCP coil with respect to permeability. (a) Resistance $R$. (b) Inductance $L$. 
Figure 5(a) shows the quality factor $Q$ of the coil. As $\mu^{\prime \prime}$ increases, the $Q$ decreases. In contrast, when $\mu^{\prime}$ increases, the $Q$ increases. $Q$ decreases as $R$ increases and improves as $L$ increases. Figures $4(\mathrm{a})$ and $4(\mathrm{~b})$ demonstrate that $Q$ has changed under the impact of $R$ and $L$.

Figure 5(b) shows the coupling coefficient $k$. A trade-off can be observed between $\mu^{\prime}$ and $k$. As $\mu^{\prime}$ increases, the coupling coefficient $k$ decreases because the magnetic flux linking the power transfer coil to the MPC coil decreases. $\mu^{\prime \prime}$ does not affect the distribution of the magnetic flux or the coupling coefficient $k$.
Figure 5(c) shows the transmission efficiency between the coils, $\eta_{\mathrm{C}}$. While the quality factor $Q$ is improved due to the increase in $\mu$, the improvement in the efficiency of the material of high permeability is low because the coupling coefficient $k$ decreases. However, the efficiency improvement is large in this case since $\mu^{\prime \prime}$ does not affect the coupling coefficient $k$. Therefore, the use of a low-loss magnetic material is optimal because the impact of $\mu^{\prime \prime}$ on the efficiency is larger than that of $\mu^{\prime}$.

(a)

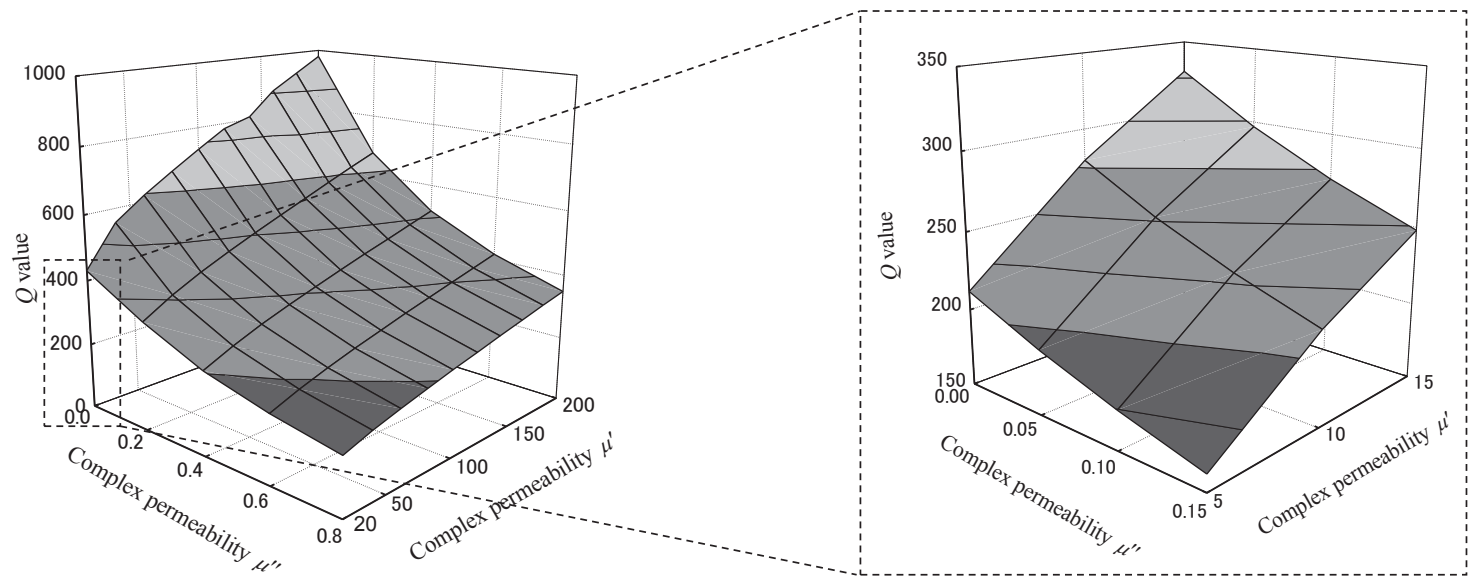

(b)

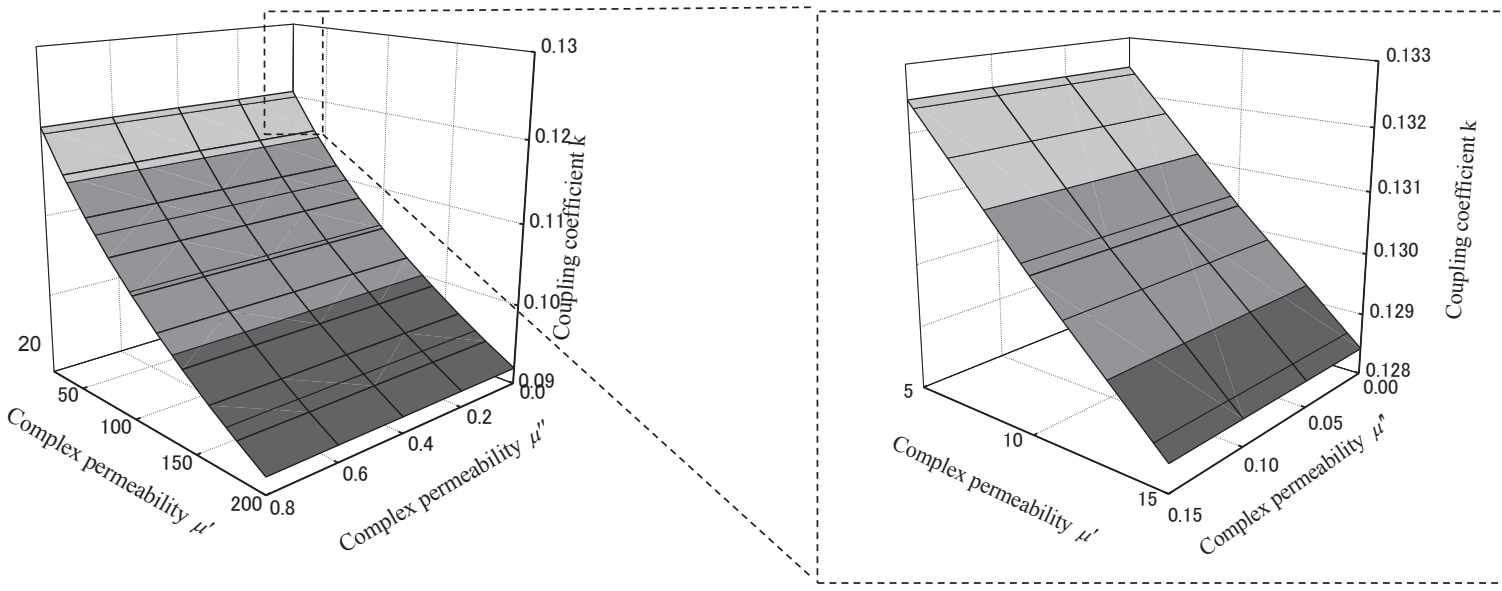

(c)

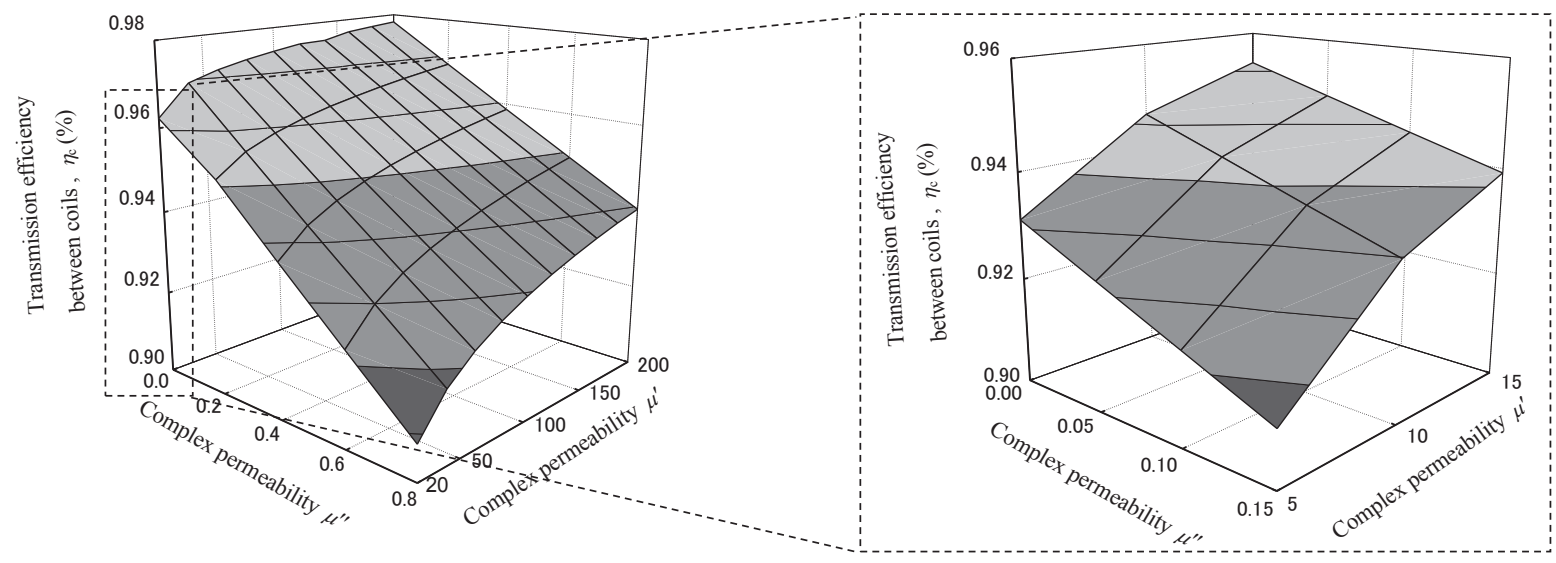

Fig. 5 Characteristics of the MCP coil with respect to permeability. (a) Quality factor $Q$. (b) Coupling coefficient $k$. (c) Transmission efficiency between coils, $\eta_{\mathrm{c}}$. 
Table 1 Specification of the magnetic material.

Amorphous metal

Volume filling rate

$57 \mathrm{Vol} \%$

Mean diameter $D_{50}$

$2.6 \mu \mathrm{m}$

Saturation magnetization $B_{\mathrm{s}}$

$0.80 \mathrm{~T}$

Complex

$$
\mu^{\prime}(f=85 \mathrm{kHz})
$$

permeability

$\mu^{\prime \prime}(f=85 \mathrm{kHz})$

0.082 frequency. The magnetic coating reduced the resistance from $85.7 \mathrm{~m} \Omega$ to $66.9 \mathrm{~m} \Omega$.

Figure 6(b) shows the inductance of the manufactured coil. The inductance is not affected by the frequency. The magnetic coating increased the inductance from $21.3 \mu \mathrm{H}$ to $22.1 \mu \mathrm{H}$. The inductance of the MCP coil was $22.1 \mu \mathrm{H}$ and that of the LCW coil was $15.1 \mu \mathrm{H}$. Note that the LCW coil had a smaller inner diameter compared to that of the AP coil and had a low inductance.
Table 1 lists the characteristics of the magnetic material used for magnetic coating. A magnetic composite material, in which an amorphous alloy powder and a silicone adhesive were mixed, was used as the magnetic material ${ }^{18}$. The ratio of the magnetic powder to the silicone adhesive in the magnetic composite material was defined as the volume filling rate, which was 57 Vol\%. The amorphous alloy contained a spherical powder with a diameter of 2.6 $\mu \mathrm{m}$. A vibrating sample magnetometer (VSM, Riken Denshi) was employed for measuring saturation magnetization $B$ s. A B-H analyzer(IWATSU, SY-8218) was used to measure the magnetic permeability. A low-loss magnetic composite material was used for magnetic coating.

\section{Characterization of the Manufactured Coil}

\subsection{Coil impedance characteristics and transmission efficiency}

Figure 6 shows the characteristics of the manufactured coil. An impedance analyzer was used for conducting the measurements. The inter-coil efficiency was calculated according to Eq. (2). The coupling coefficient $k$ was calculated from the mutual inductance $M$ product using Eq. (3). The mutual inductance $M$ was calculated from the inductance $L_{a}$ and the antiphase inductance $L_{b}$ in the in-phase series using Eq. (4).

$k=\frac{M}{\sqrt{L_{1} L_{2}}}$

$M=\frac{L_{\mathrm{a}}-L_{\mathrm{b}}}{4}$

Here, $L_{1}$ denotes the transfer coil inductance $(\mathrm{H}), L_{2}$ denotes the receiving coil inductance $(\mathrm{H}), Q_{1}$ denotes the transfer coil quality factor, and $Q_{2}$ denotes the receiving coil quality factor.

Figure 6(a) shows the resistance of the manufactured coil. As the frequency increases, the skin effect and proximity effect increase resulting in the increase in the $\mathrm{AC}$ resistance. Wireless power transmission for EVs uses the $85 \mathrm{kHz}$ band as the

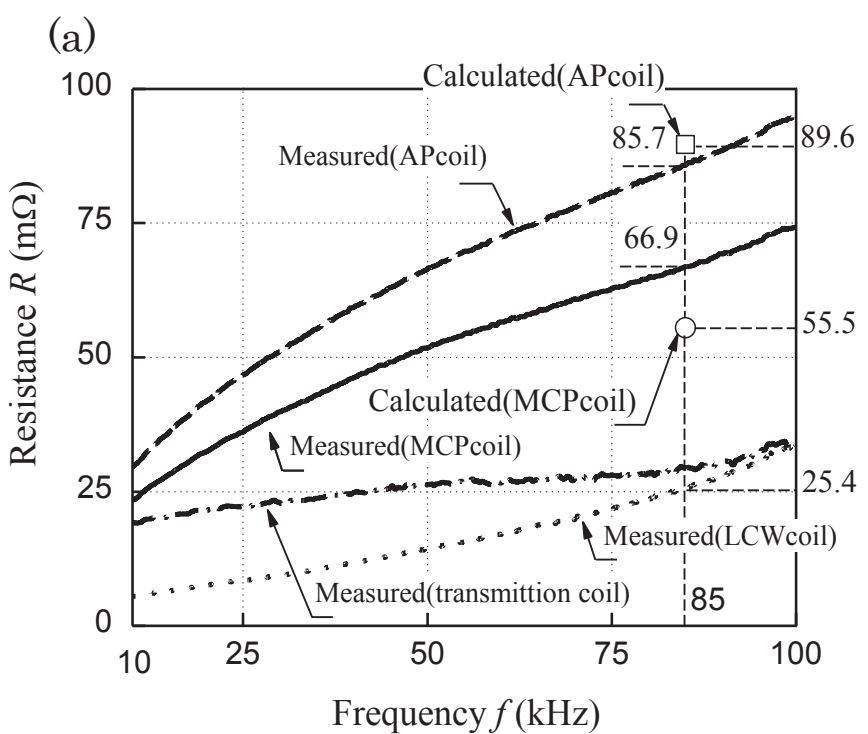

(b)

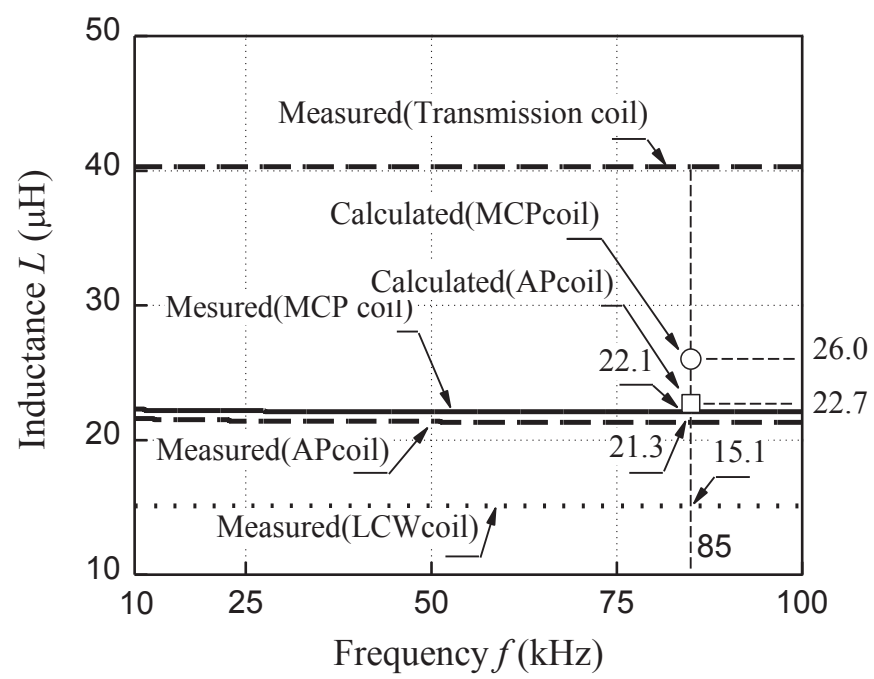

Fig. 6 Characteristics of manufactured coil. (a) Resistance $R$. (b) Inductance $L$. 
Figure 7(a) shows the quality factor $Q$ of the manufactured coil. The frequency of the quality factor is characterized by a curve that peaks at a specific value. The peak value is determined by the coil structure. Magnetic coating increased the quality factor at $85 \mathrm{kHz}$ from 135 to 176 .

(a)

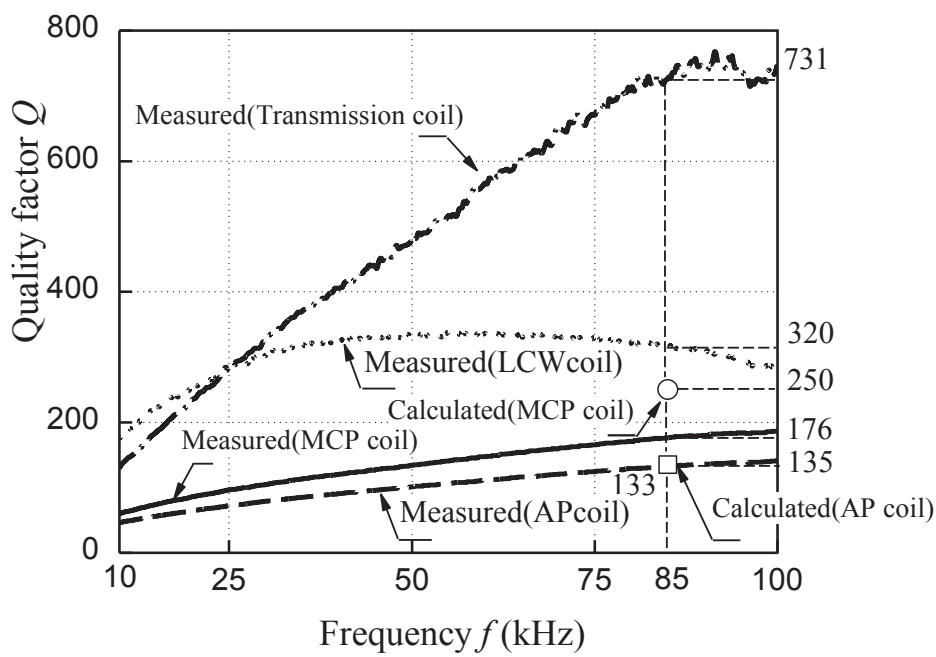

Figure 7(b) shows the coupling coefficient $k$ of the manufactured coil. The coupling coefficient is not affected by the frequency. Magnetic coating reduced the coupling coefficient at $85 \mathrm{kHz}$ from 0.161 to 0.151 .

Figure $7(\mathrm{c})$ shows the transmission efficiency between the coils. Magnetic coating improved the efficiency at $85 \mathrm{kHz}$ by $0.3 \%$. (b)

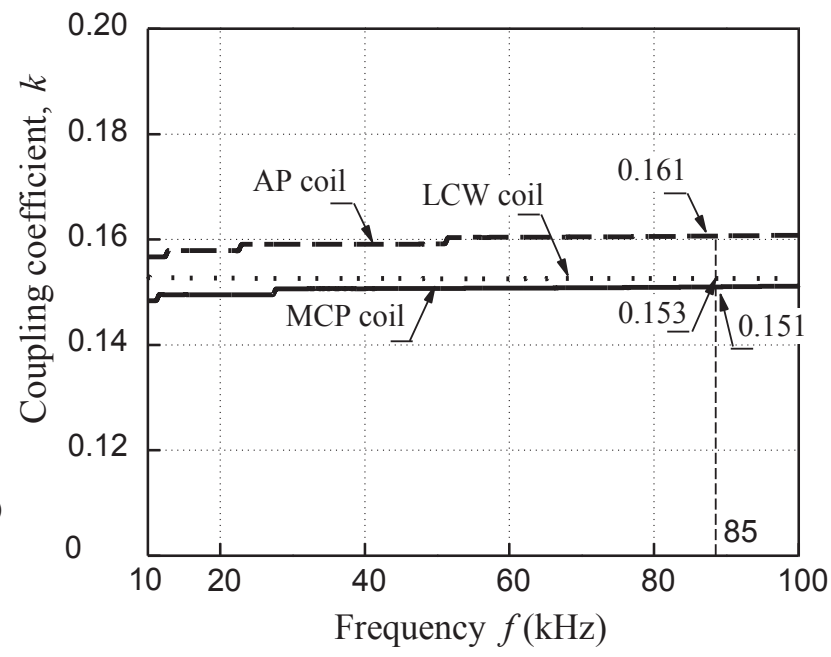

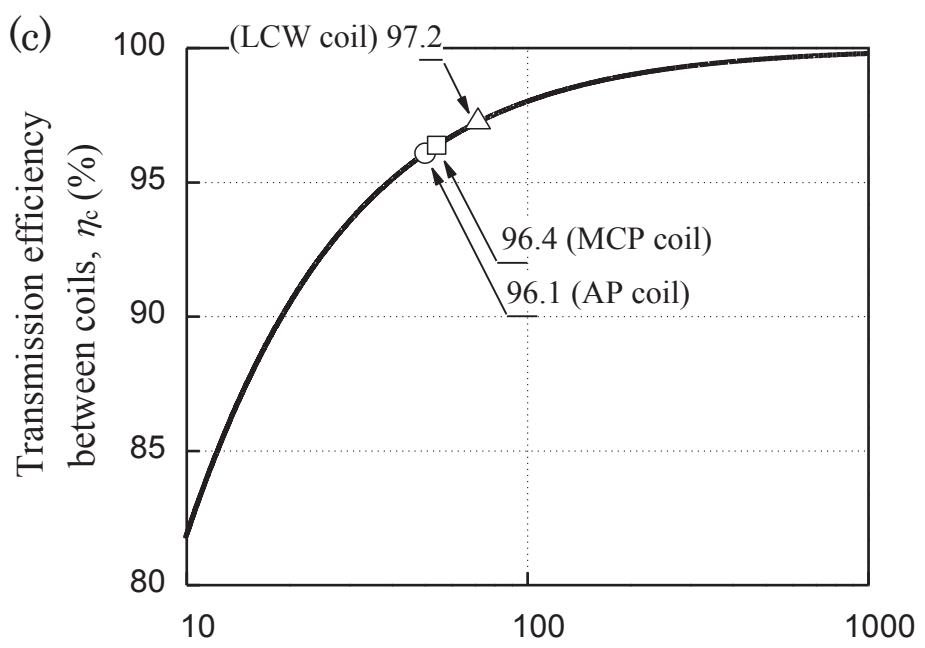

Figure of merit, $U$

Fig. 7 Characteristics of manufactured coil. (a) Quality factor Q. (b) Coupling coefficient $k$. (c) Transmission efficiency between coils, $\eta$ c. 


\subsection{Wireless power transfer experiment}

Figure 8 shows the circuit of a wireless power transmission system using the double-LCC method ${ }^{19-20)}$.

Figure 9(a) shows the transmission efficiency $\eta \mathrm{DC}$, which is indicative of the coil frequency characteristics (Eq. 5). Measurements were performed using a power analyzer (YOKOGAWA, WT 1800).

$\eta_{\mathrm{DC}}=\frac{P_{\mathrm{o}}}{P_{\mathrm{i}}} \times 100 \quad(\%)$

As shown in Figure 9(a), the maximum value $\eta_{\mathrm{DCmax}}$ of the transmission efficiency using the AP coil at the transmission distance $l=150 \mathrm{~mm}$ and the output power $P_{\text {out }}=1 \mathrm{~kW}$ was $87.7 \%$ at the frequency $f=84 \mathrm{kHz}$. The $\eta$ DCmax values were $89.0 \%$ at $f=84 \mathrm{kHz}$ for the MCP coil and $89.0 \%$ for the LCW coil at $f=87 \mathrm{kHz}$.
As depicted in Figure 9(b), the transmission efficiencies $\eta_{\mathrm{DC}}$ of the AP, MCP, and LCW coils were $88.2 \%, 89.3 \%$, and $89.4 \%$, respectively, at an output power $P_{0}=3 \mathrm{~kW}$. Hence, the magnetic coating of AP improved the transmission efficiency $\eta_{\mathrm{DC}}$ by $1.1 \%$. The difference between the transmission efficiencies $\eta_{\mathrm{DC}}$ of the MCP coil and the LCW coil was $0.1 \%$, which implies an unaltered transmission efficiency.

\subsection{Wireless power transfer coil mass}

Figure 10 denotes the mass of the manufactured coil. The total masses of the AP, MCP, and LCW coil devices were $7.69 \mathrm{~kg}, 7.84 \mathrm{~kg}$, and $9.3 \mathrm{~kg}$, respectively. This translates to a mass reduction of $17.3 \%$ for the AP coil and $15.7 \%$ for the MCP coil compared to that the mass of the LCW coil.

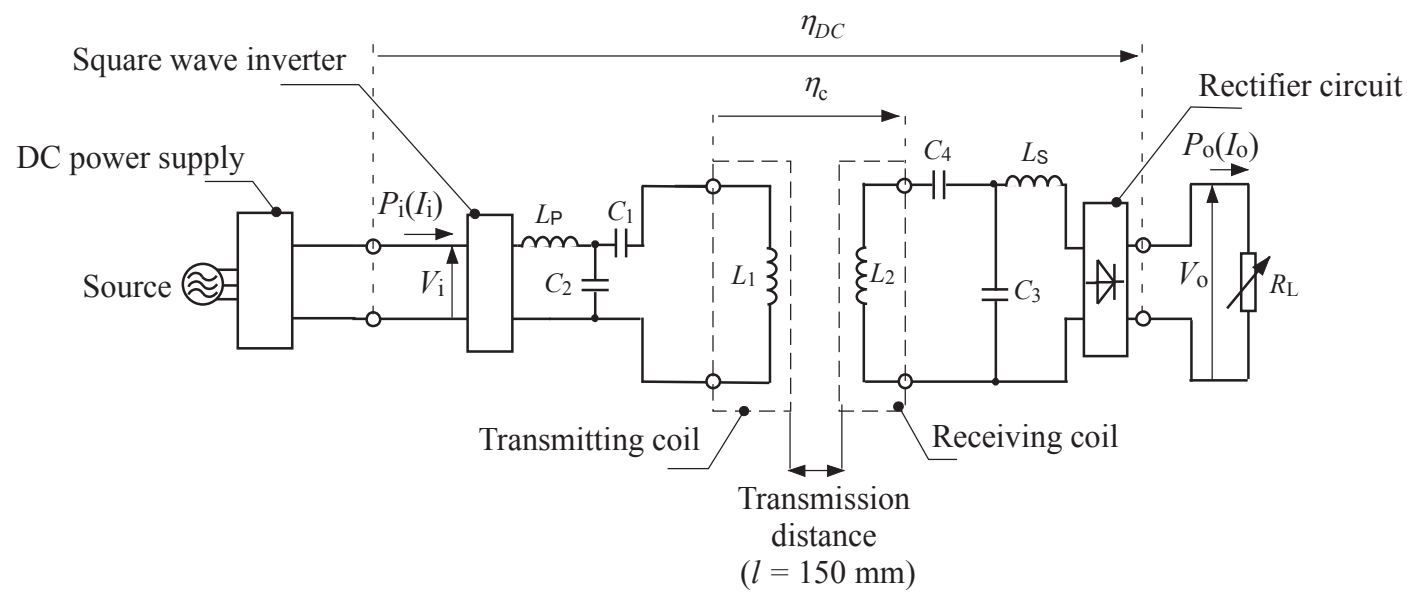

Fig. 8 Circuit for wireless power transmission $(f=85 \mathrm{kHz})$.

(a)

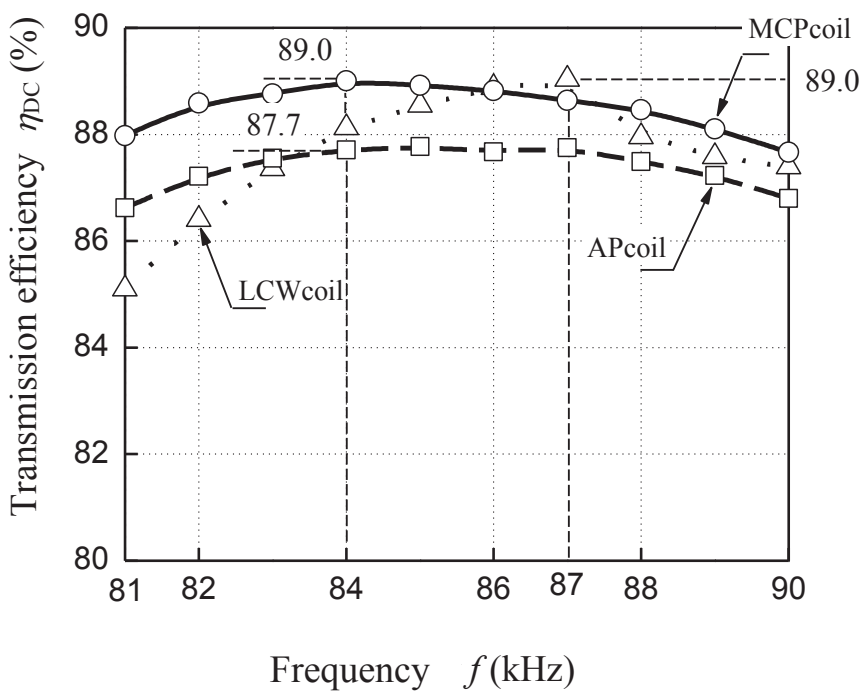

(b)

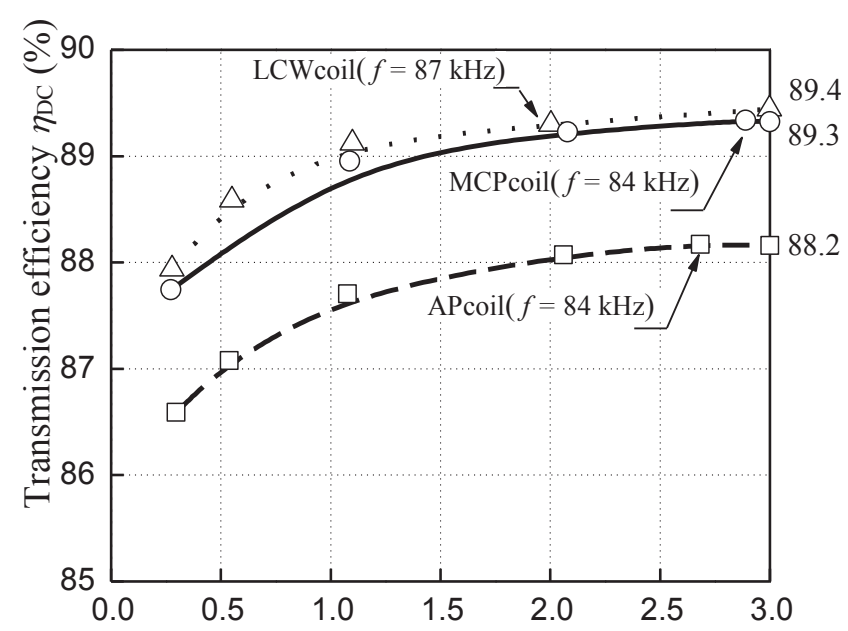

Output power $P_{\mathrm{o}}(\mathrm{kW})$

Fig. 9 Characteristics of the manufactured coil. (a)Efficiency $\eta \mathrm{DC}$ vs. frequency characteristics $\left(l=150 \mathrm{~mm}, P_{0}=1 \mathrm{~kW}\right)$. (b) Output power vs.frequency characteristics $(l=150 \mathrm{~mm})$. 


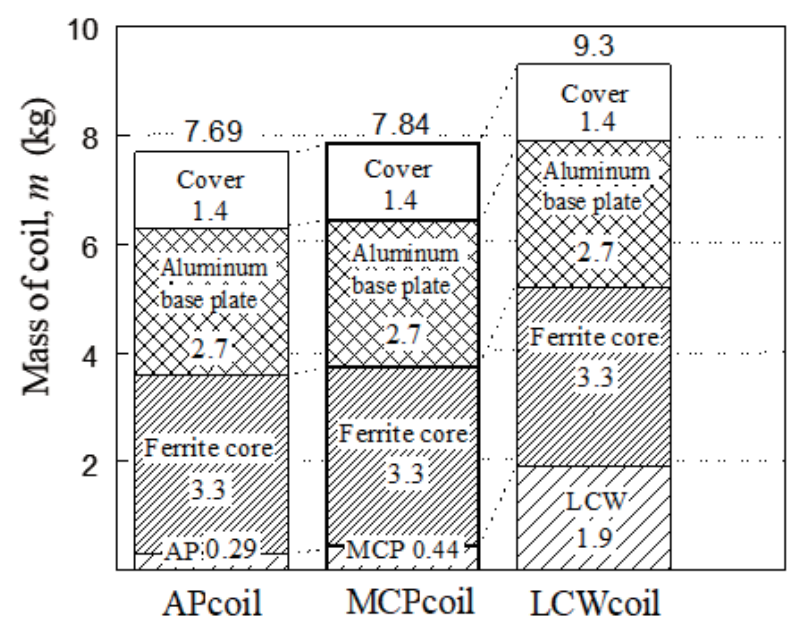

Fig. 10 Mass of the wireless power transfer coil.

The masses of the coils alone were $0.29 \mathrm{~kg}, 0.44 \mathrm{~kg}$, and $1.9 \mathrm{~kg}$ for the $\mathrm{AP}, \mathrm{MCP}$, and LCW coils, respectively. Hence, the respective masses of the $\mathrm{AP}$ and MCP coils were approximately $1 / 6$ and $1 / 4$ that of the LCW coil. Therefore, the MCP coil is considered to be advantageous because it is lightweight, easy to manufacture, and does not impede the performance.

\section{Conclusion}

FEM-based analysis revealed that the use of low-loss magnetic materials is optimal for magnetic coating.

A magnetic coating made of a magnetic composite material using a low-loss amorphous alloy powder allowed to reduce the resistance of the tested coil from $85.7 \mathrm{~m} \Omega$ to $66.9 \mathrm{~m} \Omega$. The transmission efficiency at 3 $\mathrm{kW}$ transmission improved by $1.1 \%$ compared to the transmission efficiency of the AP coil.

The transmission efficiencies of the LCW and MCP coils were observed to be equal. The mass of the MCP coil was $1 / 4$ that of the LCW coil. Therefore, the MCP coil can be considered to be advantageous because it is lightweight, easy to manufacture, and does not impede the performance.

\section{References}

1) T. Imura, H. Okabe, and Y. Hori: Proc. 2009 IEEE Vehicle Power and Propulsion Conference, Dearborn, Michigan, Sep. 7, 2009, pp. 936-942 (2009).

2) K. Throngnumchai, T. Kai, and Y. Minagawa: Proc. 2011 IEEE Energy Conversion Congress and Exposition, Phoenix, Arizona, Sep.16, 2011, pp. 843-849 (2011).

3) C. Qiu, K. T. Chau, C. Liu, and C. C. Chan: Proc. 2013 World Electric Vehicle Symposium and Exhibition (EVS27), Barcelona, Spain, Nov.17, 2013, pp.1-6 (2013).

4) Wireless Power Transfer for Light-Duty Plug-in/Electric Vehicles and Alignment Methodology, pp.82-84 (Society of Automotive Engineers, Inc., United States of America, 2019).

5) D. Barth, B. Klaus, and Thomas Leibfriend: Proc. 2017 IEEE Wireless Power Transfer Conference (WPTC), Taipei, Taiwan, May.10, 2017, pp.1-6 (2017).

6) C. R. Sllivan: IEEE Transactions on Power Electronics., 16, 281 (2001).

7) R. P. Wojda, and M. K. Kazimierczuk: IET Power Electronics., 5,257 (2012).

8) R. P. Wojda, and M. K. Kazimierczuk: IEEE Transactions on Industry Applications., 54, 3548 (2018).

9) J. Acero, R. Alonso, J. M. Burdio, L. A. Barragan, and D. Puyal: IEEE Transactions on Power Electronics., 21, 856 (2006).

10) C. Carretero: IEEE Transactions on Industrial Electronics., 64, 4474 (2017).

11) T. Mizuno, T. Ueda, S. Yachi, R. Ohtomo, and Y. Goto: IEEJ Journal of Industry Applications., 3, 35 (2014).

12) Y. Tang, H. Ma, D. J. Thrimawithana, and U. K. Madawala: Proc. 2017 IEEE PELS Workshop on Emerging Technologies: Wireless Power Transfer (WoW), Chongqing, China, May.22, 2017, pp.167-172(2017).

13) T. Yamamoto, Y. Bu, and T. Mizuno: IOS Press., 23, 486 (2016).

14) T. Yamamoto, Y. Bu, T. Mizuno, Y. Yamaguch, T. Kano: IEEJ Journal of Industry Applications., 7, 43 (2018).

15) Y. Bu, S. Endo, T. Mizuno: IEEE Trans. Magn., 54, 8401205 (2018).

16) S. Park: IEEE Trans. Magn., 54, (2018).

17) A. Kurs, A. Karalis, R. Moffatt, J. D. Joannopoulos, P. Fisher, and M. Soljacic: Science, 317, 83 (2007).

18) K. Sugimura, D. Shibamoto, N. Yabu, and T. Yamamoto., IEEE Trans. Magn., 53, (2017).

19) T. Kan, T. D. Nguyen, J. C. White, R. K. Malhan, and C. C. Mi: IEEE Trans. Power Electron., 32, 1638(2017).

20) S. Li, W. Li, J. Deng, T. D. Nguyen, C. C. Mi, IEEE Trans. Vehicular Technology, 64, 2261 (2015).

Received Jul. 09, 2019; Revised Sep. 1, 2019; Accepted Sep. 19, 2019 